\title{
DaF/DaZ und empirische Forschung: wechselnde Herausforderungen ${ }^{1}$
}

\section{Claudia Riemer}

\section{Ausgangslage}

Das Fach Deutsch als Fremdsprache hat seit den ersten Schritten seiner Konstituierung an den deutschen Universitäten eine längere Wegstrecke erfolgreich bewältigt. Das Fach hat DaF-spezifische Studiengänge entwickelt und überarbeitet und es ist jüngst dabei, den BolognaProzess zu bewältigen; es wurden in diesen Studiengängen Absolventinnen und Absolventen hervorgebracht und wissenschaftliche Nachwuchskräfte ausgebildet, von denen einige im Zuge des Generationenwechsels an den Universitäten jetzt Stellen besetzen, die zuvor noch nicht mit Wissenschaftlerinnen und Wissenschaftlern besetzt waren bzw. besetzt sein konnten, die ihre wissenschaftliche Sozialisation (zumindest in größeren Anteilen) im Fach DaF selbst durchlaufen haben und ihre wissenschaftlichen Qualifikationsarbeiten im Bereich der DaF-/ DaZ-Forschung geschrieben haben.

Es kann daher nicht mehr von DaF als einem sehr jungen Fach gesprochen werden; vielmehr gilt es, das bereits Erreichte $\mathrm{zu}$ sichern und $\mathrm{zu}$ erweitern - und das in einer Zeit, in der es gerade kleinere Fächer an den Universitäten sehr schwer haben.

\section{Problemaufriss}

Dieser Beitrag behandelt generelle Fragen und Probleme empirischer Forschung im Bereich Deutsch als Fremdsprache/Deutsch als Zweitsprache. Mit empirischer Forschung bezeichne ich Forschung, die datengeleitet und dabei systematisch und methodisch kontrolliert Erkenntnisse über die Wirklichkeit des Lehrens und Lernens von Fremdsprachen (hier: DaF) sammelt. Hierzu ist zunächst zu sagen, dass solche Forschung in unserem Fach aktiv betrieben wird. Es gibt deutlich erkennbare Fortschritte in der Breite der behandelten Themen, im Forschungsvolumen und auch in der wissenschaftlichen Reflexion der angewendeten Forschungsmethoden. Auch dass Berichte aus der empirischen Forschung einen festen Platz im Rahmen der Jahrestagungen des Fachverbands Deutsch als Fremdsprache gefunden haben und dass in den letzten Jahren immer

1 Bei diesem Beitrag handelt es sich um einen Plenumsvortrag zum Themenschwerpunkt 1: »Empirische Forschung im Bereich $\mathrm{DaF}$ « im Rahmen der 35. Jahrestagung Deutsch als Fremdsprache des Fachverbandes Deutsch als Fremdsprache (FaDaF) vom 31. Mai bis 2. Juni 2007 an der Freien Universität Berlin zum Rahmenthema »Auf neuen Wegen. Deutsch als Fremdsprache in Forschung und Praxis«. Der Beitrag wird auch in den Sammelband zu dieser Tagung in der Reihe Materialien Deutsch als Fremdsprache (MatDaF) aufgenommen. Der Tagungsband erscheint voraussichtlich im Frühjahr 2008. 
wieder auch Themenschwerpunkte eingerichtet wurden, in denen Berichte über empirische Forschungen zentral eingeplant waren, ist ein Anzeichen dafür.

Es gilt aber noch einige zentrale Herausforderungen $\mathrm{zu}$ bewältigen. Es muss ganz nüchtern konstatiert werden: Empirische Forschung im Bereich DaF/DaZ ist dadurch gekennzeichnet, dass in anderen Disziplinen erreichte Standards (z. B. in Psychologie und dabei v. a. in der pädagogischen Psychologie, in empirischer Erziehungswissenschaft und Sozialwissenschaft) in unserem Fach nicht durchgängig eingehalten werden, oft gar nicht eingehalten werden können - und dies gilt sowohl für quantitative als auch für qualitative Forschung. Ursachen liegen bekanntermaßen in den eingeschränkten Kapazitäten des Fachs an den Universitäten, in der personellen und Sachmittelausstattung von Forschungsprojekten, wenn sie überhaupt durchgeführt werden können, aber auch in fehlender oder nicht ausreichender Methodenexpertise der Forscherinnen und Forscher, die eine Entsprechung in fehlenden Modulen zu Forschungsmethoden und fehlender Forschungspraxis im Rahmen der DaF-/ DaZ-Studiengänge finden.

Meine Ausgangsthese lautet, dass das Fach Deutsch als Fremdsprache momentan in eine Phase eintritt bzw. sich schon längst befindet, in der Forschung, und dabei insbesondere forschungsmethodologisch kontrollierte empirische Forschung, massiv die Zukunft des Fachs als akademische Disziplin bestimmen wird. Und damit meine ich sowohl empirische Forschung, die im Bereich DaF/DaZ selbst durchgeführt wird, als auch empirische Forschung, die in anderen Disziplinen geleistet wird und deren Ergebnisse uns (teilweise ganz direkt) betreffen

Welche empirische Studie, welche Studien wurden in den letzten Jahren in der gesellschaftlichen Öffentlichkeit und auch im Rahmen der DaF-Tagungen am häufigsten zitiert? Welche Studien haben vielleicht sogar Schockwellen ausgelöst? Welche Studien haben daraufhin auch in unserem Fach Bewegungen in Gang gesetzt, eine Fokusverschiebung von DaF zu DaZ - welche Studien haben dem Fach damit auch höhere Relevanz und Aufmerksamkeit in der Öffentlichkeit, der Politik, an den Universitäten beschert? Eine Antwort ist: die PISA-Studien und die Erkenntnis, dass Kinder und Jugendliche mit Zuwanderungshintergrund und dabei insbesondere diejenigen, die in Deutschland nicht als Seiteneinsteiger, sondern von Beginn an das schulische Bildungssystem durchlaufen - bei der Entwicklung spezifischer sprachlicher Kompetenzen, die für erfolgreiche Bildungskarrieren zentral sind, massiv im Nachteil sind. Waren aber DaF-/DaZler an der Konzeption der Studien und an den Analysen beteiligt - gibt es doch seit vielen Jahren DaF-/DaZ-Fachvertreterinnen und -vertreter, die mit Nachdruck auf die Notwendigkeit der systematischen Förderung von Kindern und Jugendlichen mit Migrationshintergrund aufmerksam gemacht haben? Nein.

In den letzten Jahren haben wir außerdem (auch im Rahmen der Jahrestagungen) viel über die Integrationskurse diskutiert - und werden dies weiter tun. Vertreter von DaF-Lehrstühlen sind in der das BAMF beratenden Bewertungskommission vertreten. Eine empirische Evaluationsstudie der Integrationskurse wurde vom BMI ausgeschrieben, schließlich in Auftrag gegeben und dann Anfang dieses Jahres veröffentlicht. Laut BAMF-Internetauftritt war es Ziel der Studie

»dabei nicht, die politischen Vorgaben zu hinterfragen, sondern vielmehr deren Umsetzung $\mathrm{zu}$ analysieren. Im Fokus standen die Bereiche >Verfahrenseffizienz‘, >Finanzierung < und >Methodik/Didaktik « (www.bamf.de, 30.1.2007). 
Haben DaF-/DaZler sich massiv um die ausgeschriebene Studie beworben, haben sie die Studie durchgeführt - oder bescheidener gefragt: War DaF-/DaZ-Kompetenz im Team vertreten, das die Studie durchgeführt hat? Die Antwort lautet erneut: nein. Das kommerzielle Management-Beratungsunternehmen, das die Studie durchgeführt hat, äußert sich dazu im Abschlussbericht indirekt folgendermaßen:

»Um der Komplexität des Untersuchungsgegenstandes Rechnung zu tragen, stellte Rambøll Management ein Evaluationsteam aus unterschiedlichen wissenschaftlichen Disziplinen zusammen. Durch diesen multidisziplinären Ansatz waren für jeden Untersuchungsaspekt spezifische Fachkompetenzen vorhanden. Im Evaluationsteam waren die folgenden wissenschaftlichen Hintergründe präsent:

- Politologie

- Jura

- Betriebswirtschaftslehre

- Volkswirtschaftslehre

- Internationales Informationsmanagement

- Erziehungswissenschaften

- Soziologie/Statistik

- Psychologie

- Geographie

- Informatik«

(Bundesministerium des Innern 2006: 6)

Solche empirische Forschung muss zunächst einmal als von außen an das Fach herangetragene Herausforderung wahrgenommen werden. Ich frage aber hier zu Beginn meines Beitrags ganz offen und ungeschützt: Ist das Fach DaF überhaupt hinreichend darauf vorbereitet, sich in kleine und große empirische Studien im Rahmen der Bildungsforschung angemessen einzubringen, in Studien, bei denen das Lernen und Lehren von Deutsch als Fremd- oder Zweitsprache reliabel und valide evaluiert werden soll, Studien, in denen die Wirksamkeit entwickelter Konzeptionen, eingesetzter Methoden und Materialien methodisch kontrolliert und systematisch überprüft wird, in denen Entwicklungen bei Aneignungsprozessen $\mathrm{DaF} / \mathrm{DaZ}$ umfassend und mehrperspektivisch erforscht werden? Wenn wir solche Fragen nicht überzeugend bejahen können, wenn wir sie auch gegenüber potenziellen Forschungsförderern, Auftraggebern von Forschung und in interdisziplinären Forschergruppen nicht überzeugend bejahen können, haben wir ein Problem. Wir - damit meine ich die Praxis und die universitären DaF-/DaZ-Forschungsbereiche. Betroffen ist die Praxis, die möglicherweise mit den direkten Folgen solcher Forschung leben muss, z. B. im Rahmen von Sprachförderangeboten, die nicht angemessen ausgestattet sind und sich möglicherweise von Fachfremden vorhalten lassen müssen, dass $u$. a. der Unterricht qualitativ verbessert werden muss, wenn die Ergebnisse einer Sprachfördermaßnahme nicht den Erwartungen entsprechen (wenn z.B. das Erreichen einer Niveaustufe B1 von einem großen Anteil von Lernenden in einer Fördermaßnahme nicht erreicht werden kann). Die universitären DaF-Bereiche hängen auch hinsichtlich ihrer Eigenständigkeit in Form von eigenen Studiengängen und Promotionsmöglichkeiten und der damit möglichen Ausbildung auch des wissenschaftlichen Nachwuchses von einer erfolgreichen Forschungsentwicklung ab. Die Zeit der Freiheit in Lehre und Forschung scheint endgültig vorbei zu sein. Einbindbarkeit von Neuberufenen in größere Forschungsverbünde an den jeweiligen Universitäten (und in denen gibt es im schlimmsten Fall keine oder im Normalfall nur wenige andere DaF-/ DaZler oder Fremdsprachendidaktiker anderer Philologien) ist zunehmend zum wichtigen Kriterium in Berufungskommissionen und Berufungsentscheidungen von Universitätspräsidien geworden; individuelle Forschungsleistungen gelten schon jetzt mancherorts als zweite 
Wahl. Hinzu kommt: Gute Forschungsleistungen über die Einwerbung von Forschungsdrittmitteln $\mathrm{zu}$ beweisen, ist bereits heute wichtig und im Rahmen von Zielvereinbarungen und Besoldungsverhandlungen ein zentraler Punkt. Der Druck wird mit Sicherheit zukünftig noch zunehmen (Stichwort: Exzellenzinitiative, in deren Folge die DFG z. B. Gutachter darauf hinweist, dass nur mehr solche Forscherpersönlichkeiten förderwürdig sind, die »national führend « oder besser noch »international sichtbar « sind - schlechte Chancen für Neueinsteiger, für Neuberufene. Wer an der Aufrechterhaltung und für den Ausbau von Deutsch als Fremdsprache im Fächerkanon der Universitäten mitarbeitet, muss sich dieser Herausforderung nichtsdestotrotz stellen.

Ich möchte die Relevanz empirischer Forschung nicht allein - wie es in der Vergangenheit oft getan wurde - auf den Bereich der fremdsprachenlehr-/-lernwissenschaftlichen Ausrichtung des Fachs beziehen. Die Unterscheidung von unterschiedlichen Ausrichtungen (lehr-/ lernwissenschaftlich oder didaktisch-methodisch, linguistisch, literaturwissenschaftlich, kulturwissenschaftlich) hatte meines Erachtens heuristischen Wert in der Phase der akademischen Konstituierung des Faches - sie ist heute aber nicht mehr zielführend. Zukünftig sollte eher das Verhältnis dieser Ausrichtungen zueinander im Rahmen einer integrativen Fachkonzeption im Zentrum stehen. So kann auch kein Studiengang, der ernsthaft eine umfassende Ausbildung in Deutsch als Fremd- und Zweitsprache anstrebt, ohne entsprechende Studienelemente aus den unterschiedlichen Bereichen auskommen. Die federführend vom Fachverband Deutsch als Fremdsprache benannten Kernbereiche, die in einem Bachelor-/Masterstudiengang $\mathrm{zu}$ berücksichtigen sind, sprechen hier eine deutliche Sprache, obwohl sie eher bescheiden formuliert sind (vgl. Fachverband Deutsch als Fremdsprache 2006). Es wird weiter unterschiedliche Forschungsschwerpunkte an den universitären DaF-Abteilungen geben; hier ist angesichts des hohen Forschungsbedarfs in allen Bereichen Vielfalt sogar ausdrücklich zu begrüßen und zu fördern. Die auch empirische Ausrichtung dieser Forschungsschwerpunkte mit Nachdruck anzustreben bzw. - wenn bereits in Ansätzen vorhanden - diese auszubauen und weiterzuentwickeln, halte ich jedoch für unverzichtbar, wenn die wissenschaftliche Beschäftigung mit deutscher Sprache, Kultur und Literatur im für das Fach konstitutiven Verbund mit damit verbundenen fremd-/zweitsprachlichen und fremdkulturellen/interkulturellen Prozessen der Aneignung und Verwendung erfolgt. Wenn ich im Folgenden also von Fremdsprachenlernen und -lehren spreche, schließt dies immer auch Prozesse des kulturellen Lernens ein.

Forschung im Bereich DaF/DaZ kann dabei anknüpfen an Entwicklungen in der internationalen Fremdsprachenforschung (insbesondere Applied Linguistics, Second Language Acquisition Research, Bilingualismusforschung) und in der deutschen Fremdsprachenforschung (Sprachlehr- und -lernforschung, empirische Fremdsprachendidaktik).

Ich möchte kurz ausholen, warum zunächst im Bereich der lehr-/lernwissenschaftlichen Ausrichtung des Fachs empirische Forschung vorangetrieben wurde. Dies hatte zu tun mit der Entwicklung der Sprachlehr-/-lernforschung in Deutschland und der dann schrittweise erfolgten Umorientierung der Fremdsprachendidaktik, für die die Erforschung des Fremdsprachenunterrichts und dabei insbesondere die empirische Untersuchung seiner prozessualen 
Lehr- und Lernaspekte übergeordnetes Ziel ist. Angestrebt wird dabei,

»die begründete Konsolidierung bzw. Veränderung konkreter Formen des Lehrens und Lernens fremder Sprachen zu bewirken « (Bausch/Christ/Krumm ${ }^{4}$ 2003: 4).

Eine solche Forschung sei in ihren Arbeitsweisen aufgefordert,

»die Forschungsmethodik so anzulegen, dass sie Probleme aus der Praxis aufgreift, der systematischen und integrativen Forschung zuführt und wieder in die Praxis einbringt, sei es in Form von Bestätigungen für gewohntes Unterrichtsverhalten, sei es als Empfehlung bzw. Handlungsalternative für eine begründete Veränderung desselben " (Bausch/Christ/Krumm ${ }^{4}$ 2003: 4).

Diesem Credo der Sprachlehrforschung entsprechend wurde empirische Forschung in den letzten 20, 30 Jahren gefordert und gefördert, in Teilen erfolgreich geleistet - gleichzeitig aber auch vielfach geschmäht und insgesamt noch viel zu wenig bearbeitet.

Eines wurde bis heute versäumt: parallel dazu die forschungsmethodische Ausbildung in den Studiengängen ausreichend $\mathrm{zu}$ berücksichtigen, geschweige denn $\mathrm{zu}$ verankern. Es entstand die für forschungsinteressierte und qualifikationswillige Studierende und wissenschaftliche Nachwuchskräfte unbefriedigende Situation, dass sie sich viele wichtige Grundlagen der empirischen Forschungsmethodik vorwiegend im (teils betreuten, teils unbetreuten) Selbststudium aneignen und Unterstützung außerhalb ihres Fachs suchen müssen, was nicht immer gelingt - ich spreche hier auch aus eigener Erfahrung. Viele, die ihre wissenschaftliche Sozialisation im Fach DaF durchlaufen haben und nicht etwa als Seiteneinsteiger aus der Psychologie oder Soziologie zu DaF stoßen, müssen bei null oder nahe null anfangen, wenn sie ein empirisches Forschungsprojekt anstreben, was die Wahl von z.B. anspruchsvollen multivariaten quantitativen Designs meist von vornherein ausschließt. Auch dass häufig qualitative Designs ausgewählt werden, kann darauf zurückgeführt werden; außerdem darauf, dass interpretative Verfahren der Datenanalyse an philologisch-literaturwissenschaftlich-hermeneutische Verfahren anknüpfen können, mit denen viele DaFler im Laufe des Studiums zumindest ansatzweise in Berührung kommen. Wir benötigen in unserem Bereich aber qualitative und quantitative Forschung, um zentrale Fragestellungen bearbeiten $\mathrm{zu}$ können. Im Folgenden erlaube ich mir daher einen knappen Exkurs, der die zentralen Charakteristika beider Paradigmen beleuchtet.

\section{Qualitative und quantitative For- schung}

Das qualitative und das quantitative Forschungsparadigma implizieren jeweils unterschiedliche Voraussetzungen und Ziele, gehen von unterschiedlichen zugrunde gelegten Menschenbildern und Vorstellungen über die Forschungsorganisation und -durchführung aus und verlangen Daten, die nach unterschiedlichen Maximen erhoben, aufbereitet und analysiert werden müssen. Dies führt innerhalb der jeweiligen Paradigmen zu Forschungsergebnissen mit unterschiedlichem Erkenntnisanspruch. Die von Grotjahn (1987) präferierte Verwendung der Termini "explorativ-interpretativ « bzw. "analytisch-nomologisch « als Bezeichnung dieser beiden Forschungsausrichtungen benennt die zentralen Merkmale beider Methodologien. Beide Ansätze wurden lange Zeit als sich gegenseitig ausschließende Paradigmen interpretiert, was zu Lagerbildungen führte. Heute ist eher ein Zustand friedlicher Koexistenz eingetreten, wobei aber nicht verkannt werden darf, dass trotz allseitiger Proklamation der Berechtigung beider For- 
schungsrichtungen und des Plädoyers für eine Verbindung von beiden im Rahmen von hybriden Methodologien Vertreter der jeweiligen Ausrichtung gern die Arbeiten und Entwicklungen der anderen Ausrichtung ignorieren.

Hinsichtlich einer Gegenüberstellung wichtiger Merkmale beider Paradigmen halte ich mich kurz: Auf der Ebene der allgemeinen Zielsetzung von Forschungen stehen sich Verstehen auf der qualitativen Seite und auf der quantitativen Seite Deskription und Erklären menschlichen Verhaltens gegenüber. Qualitative Forschung verlangt einen empathischen Nachvollzug aus der Perspektive der Untersuchungsteilnehmer und bedingt ein komplexes elaborativ-prospektives Menschenbild, wobei das Gesamtfeld als Informationslieferant zur Verfügung steht. (Zur Menschenbilddiskussion in der Fremdsprachenforschung vgl. Grotjahn 2005b.) Hypothesen und Theorien sollen dabei erst während des Forschungsprozesses durch das interpretative Auffinden wiederkehrender Muster erschlossen werden (qualitative Forschung als theoriegenerierende Forschung), wobei ausgeprägte Vorstrukturierungen des Untersuchungsfeldes (z. B. Standardisierungen in der Datenerhebung oder vorrangige Berücksichtigung von Vorwissen in der Entwicklung kategorialer Systeme) weitgehend vermieden werden sollen. Es interessieren nicht allein die Produkte menschlichen Verhaltens, sondern vor allem die Prozesse, die zu ihnen führen. Das Untersuchungsfeld soll so weit wie möglich natürlich belassen sein, dies heißt konkret: Soll z. B. gesteuerter Fremdsprachenerwerb verstanden werden, so sind die Daten aus dem Kontext real stattfindenden Fremdsprachenunterrichts zu gewinnen und nicht in speziell eingerichteten laborähnlichen Handlungsräumen mit je eigenen Konstellationen. Dabei sollen möglichst tiefgründige, reichhaltige
Daten (rich data) erhoben werden. Dies bedeutet andererseits, dass es aus forschungsökonomischen Gründen selten möglich ist, größere Probandengruppen $\mathrm{zu}$ erfassen und dass damit qualitative Forschungen zumeist in Form von Fallstudien organisiert sind, die auf eine breite Generalisierung der Befunde verzichten müssen. Oben erwähnte Parameter wie »Verstehen « oder »interpretativ« deuten darauf hin, dass qualitative Studien weniger dem Gütekriterium der $\mathrm{Ob}$ jektivität entsprechen können; hierfür wurden Gütekriterien wie »Nachvollziehbarkeit« und »Intersubjektivität« bzw. »reflektierte Subjektivität« eingeführt (vgl. Arbeitsgruppe Fremdsprachenerwerb Bielefeld 1996, Steinke 1999). Noch relativ wenig ist in der qualitativen Fremdsprachenforschung die Zusammenstellung von Untersuchungsgruppen/Auswahl von Fällen diskutiert worden, was meines Erachtens dringend notwendig wäre. Denn die mit der Wahl eines qualitativen Forschungsdesigns verbundenen notwendigen Entscheidungen für ein bestimmtes Lehr-/Lernumfeld (Alter, Ausgangssprache, L2-Kompetenz, Unterrichtskontext, Lernziele etc.) inklusive Verzicht auf größere Probandengruppen (und repräsentative Stichproben) dürfen nicht in Beliebigkeit bzw. Zufälligkeit der Auswahl der Forschungssubjekte resultieren.

Quantitative Forschung dagegen strebt generalisierbare Gesetzmäßigkeiten an. Es ist anzumerken, dass dieses Paradigma - wohl auch durch seine erwiesene Effizienz innerhalb naturwissenschaftlicher Forschung und den davon abgeleiteten Anspruch auf >Wissenschaftlichkeit $<-$ besonders in den letzten 50 Jahren innerhalb sozialwissenschaftlicher Forschung und in der fremdsprachenerwerbsspezifischen Forschung angloamerikanischer Ausrichtung sehr einflussreich war und weiter ist. Im Rahmen 
dieses Forschungsparadigmas sollen aus der Außenperspektive interessierende Untersuchungsobjekte erklärt werden, d.h. auf exakt zu spezifizierende Bedingungen zurückgeführt werden. Aufgestellte Hypothesen sollen dabei mit Hilfe empirischer Untersuchungen verifiziert oder falsifiziert werden. Dafür ist es unabdingbar, Teilbereiche des Untersuchungsfeldes $\mathrm{zu}$ isolieren und zu kontrollieren, d.h. externe Einflüsse und weitere intervenierende Variablen auf die interessierenden Untersuchungsgegenstände müssen hinreichend ausgeschaltet werden. Dies erfordert eine Manipulation des Untersuchungsfeldes, sorgfältige $\mathrm{Zu}$ sammenstellung der Probandengruppen und standardisierte Datenerhebungsverfahren (am besten gelingt dies innerhalb eines Experimentes in einer dafür geschaffenen Laborsituation). Erhoben werden sollen harte $-d$. h. reliable und replizierbare - Daten, die mit Hilfe teststatistisch überprüfter Instrumentarien elizitiert werden. Dies ist allerdings mit einem Verlust an Tiefgründigkeit und Natürlichkeit verbunden. Vonseiten qualitativer Forscher ist deshalb der Vorwurf erhoben worden, dass die Künstlichkeit der Datenerhebung die ökologische Validität der gewonnenen empirischen Befunde und damit gerade die angestrebte Generalisierbarkeit einschränkt.

In den letzten Jahren hat sich (nicht nur) in der Fremdsprachenforschung der Standpunkt durchgesetzt, dass der qualitative und der quantitative Forschungsansatz nicht als Paradigmen anzusehen sind, sondern vielmehr Erkenntnisinteressen und Untersuchungsgegenstände die Wahl spezifischer Forschungsdesigns begründen sollten. Für die Auswahl eines stärker qualitativ oder stärker quantitativ orientierten Forschungsdesigns ist danach u. a. entscheidend, ob zum Zeitpunkt des angestrebten Forschungsprojekts der Wissensstand zum spezifischen
Gegenstand eine begründete Hypothesenprüfung erlaubt oder es sinnvoller ist, mögliche Vorannahmen und Hypothesen über das Untersuchungsfeld erst einmal empirisch zu gewinnen.

Parallel dazu kann man beobachten, dass fremdsprachenspezifische Forschungen selten die Ideale des qualitativen oder des quantitativen Ansatzes erfüllen können. So gibt es selten in quantitativ-deskriptiven Studien ausreichend große und durch Zufallsauswahl zusammengestellte Untersuchungsgruppen. Auch genuine Experimente sind relativ selten, häufiger sind Quasi-Experimente, die etwa Bedingungen wie die Existenz zweier vergleichbarer Probandengruppen oder die Zufallsverteilung der Probanden auf Experiment- und Kontrollgruppe nicht erfüllen. Auch reine Feldforschung, die auf teilnehmender Beobachtung beruht, ist innerhalb fremdsprachenspezifischer Forschung kaum repräsentiert. Bei näherer Betrachtung der oben angedeuteten einfachen Gegenüberstellungen zwischen qualitativer und quantitativer Forschung lassen sich diese auch nicht aufrechterhalten. $\mathrm{Ob}$ Hypothesen generiert oder getestet werden sollen, ob induktiv oder deduktiv vorgegangen wird, beide Vorgehensweisen greifen auf Vorwissen zurück. Qualitative Forschung hat sich damit vor allem auf der Ebene der Datenanalyse auseinanderzusetzen, quantitative Forschung dagegen eher auf den Ebenen der Datenerhebung und -aufbereitung, wenn etwa kategoriale Raster für Fragebögen konzipiert und ausgewertet werden. Allerdings bleiben Unterschiede bestehen, die in den mit qualitativer und quantitativer Forschung verbundenen unterschiedlichen Vorgehensweisen und Forschungsstrategien begründet sind. Auf der einen Seite steht etwa das notwendige Festhalten an einem strikten Forschungsplan zur Erhebung vergleichbarer Daten und de- 
ren systematische sowie vorweg festgelegte Analyse im Rahmen quantitativer Forschung, auf der anderen Seite bei qualitativer Forschung die Notwendigkeit eines stärker offenen und hinsichtlich sich ergebender Bedingungen des Untersuchungsfelds anpassbaren Forschungsplans, der sich z. B. in sukzessiver Probandenauswahl und modifizierten Erhebungsverfahren niederschlägt.

\section{Akzeptanzprobleme}

Zwar kann in den letzten Jahren insgesamt ein Anstieg im Volumen empirischer Forschung v.a. im Rahmen von Qualifikationsarbeiten des wissenschaftlichen Nachwuchses beobachtet werden, der auch damit zu tun hat, dass eine empirische Erarbeitung vielerorts von den Betreuern solcher Arbeiten verlangt wurde und wird. Trotzdem (oder vielleicht sogar als Folge davon?) ist heute teilweise offen $\mathrm{zu}$ hören, dass >die Sprachlehr-/-lernforschung ihre programmatischen Versprechungen nicht eingehalten haber, teilweise wird getuschelt, dass >die ganze Empirie doch nichts oder nur wenig gebracht habe<, dass die Arbeiten oft methodisch nicht überzeugen können und man besser wieder $\mathrm{zu}$ >hermeneutischer Forschung (was immer auch darunter verstanden werden mag) zurückkehren könne. Nicht nur in der fremdsprachenunterrichtlichen Praxis herrscht Zweifel, ob die beschworene angestrebte empirische Begründung spezifischer fremdsprachendidaktischer Handlungsstrategien oder allgemeiner Prinzipien nicht doch eher die wissenschaftliche Deskription des bereits Bekannten oder spezifischer Sonderfälle darstelle, und es gibt auch die Meinung, dass solche Forschung die >wahren Probleme nicht löse. Solche Einschätzungen werden häufig dann laut, wenn Fragen zur Wirksamkeit bzw. Effektivität spezifischer Instruktionsformen, Lernarrange- ments und Lernmaterialien gestellt werden und aus der Forschungsperspektive nicht zufriedenstellend beantwortet werden können.

Was ist los? Ist empirische Forschung im Bereich DaF/DaZ und allgemein in der deutschen Fremdsprachenforschung in eine Sackgasse geraten, die uns nicht zu den goldenen Töpfen akademischer und gesellschaftlicher Anerkennung führt? Warum fehlt Wirkungsforschung weitgehend, die etwas Profundes darüber aussagen kann, ob, wann und wie Fremdsprachenunterricht in seinen unterschiedlichen Ausprägungen Lernen bewirkt? Dies ist zweifelsohne ein Forschungsfeld, das besonders intensive Bearbeitung benötigt, von Bildungspolitikern und Praktikern gefordert wird, aber aus unterschiedlichen Gründen zu wenig angegangen wird. Als exemplarisches Beispiel möchte ich hier lediglich die weitgehend fehlende Lehrmaterialwirkungsforschung nennen, die nicht erst seit dem mit der Adaption von Lernmaterialien an den Europäischen Referenzrahmen boomenden Lehrwerkmarkt ein dringliches Desiderat darstellt. DaF gilt das habe ich immer wieder bewundernd von Kolleginnen und Kollegen aus der Englisch- oder Französischdidaktik gehört - als Vorreiter in der Lehrwerkentwicklung - aber leider nicht im Bereich der Lehrmaterialwirkungsforschung. Solche und andere Defizite können dazu führen, dass - aus der Außenperspektive betrachtet - von empirischen Forschern möglicherweise immer gerade das $>$ Falsche< erforscht wird.

Akzeptanzprobleme von Forschung bei Praktikern mögen eine Ursache darin haben, dass das Postulat, zu begründeten Handlungsstrategien im Fremdsprachenunterricht zu gelangen, häufig in empirischen Studien nicht oder nur in kleinen Ausschnitten erreicht werden kann. Häufig müssen sich solche Arbeiten auf allge- 
meine Prinzipien bzw. Ziele der Sensibilisierung von Rezipienten für ausgewählte Bereiche des Lehrens und Lernens beschränken. Eine Folge enttäuschter Erwartungen auf Seiten der Lehrenden, von Forscherseite konkrete Vorschläge zur Umsetzung der empirisch gewonnenen Ergebnisse für die eigene didaktische Praxis zu erhalten, kann sein, dass der Dialog mit der Forschung ganz aufgegeben wird (vgl. zu Störfeldern zwischen Theorie und Praxis auch Aguado/Riemer 2000) bzw. auf die Phase der Ausbildung und punktuelle Weiterbildung beschränkt und ohne nachhaltige Auswirkungen bleibt. Die Untersuchung von Lehrerhaltungen (vgl. exemplarisch Caspari 2003) hat bestätigt, dass überwiegend eigene frühere Sprachlernerfahrungen, vorwissenschaftliche Überzeugungen sowie lehr-/lernkontextspezifische Erwägungen unterrichtliches Handeln von Lehrenden leiten. Eine letztes Jahr in der Fachzeitschrift Modern Language Journal publizierte Studie von Rankin/Becker (2006) mit dem Titel: »Does reading the research make a difference? A case study of teacher growth in FL German « kommt zum Schluss, dass die Rezeption von Forschungsergebnissen das Handeln eines (noch unerfahrenen) Lehrers zwar nachweislich beeinflusst, dies geschieht aber durch einen Filter vorhandener Einstellungen und Lernerfahrungen, ein einfacher Transfer von Wissen in Handeln ist nicht gegeben - es muss in den persönlichen Rahmen eingepasst und v. a. gut reflektiert werden. Untersucht wird in dieser Studie das recht gut erforschte mündliche Korrekturverhalten von Lehrern, was ein Forschungsbereich ist, dem wahrlich nicht vorgeworfen werden kann, praxisfern und implikationslos zu sein.

Aktionsforschung, die begründet etwas zur Effektivität von Fremdsprachenlehre sagen könnte, ist in unserem Bereich kaum ausgeprägt (vgl. diesbezügliche Überlegungen in Riemer 2002). Leider! Denn Aktionsforschung wird insbesondere das Potenzial zugeschrieben, dass aus der konkreten Unterrichtswirklichkeit entnommene Fragestellungen nach ihrer systematischen Analyse in Handlungsstrategien für die Unterrichtsgestaltung überführt werden, die sofort in ihren Auswirkungen reanalysiert werden können und dann zu weiteren Modifikationen führen können. Ursachen für das Fehlen solcher Forschung (v. a. in größeren und von der Fachöffentlichkeit wahrgenommenen Zusammenhängen) mögen darin liegen, dass solche Aktionsforschung hierzulande, anders als z. B. in Großbritannien und den USA, auf keine Tradition zurückblicken kann und auch keinen entscheidenden Einfluss auf curriculare Entwicklungen nehmen konnte. Hinzu kommen allgemeine Probleme, die Forschungsinteressen von Lehrenden massiv beeinträchtigen, wie Befristungen und schlechte Bezahlung von Lehrtätigkeiten, Zeitmangel, Mangel an Unterstützung (durch Kollegen, durch die Institution) und Mangel an Erfahrung mit Forschungsprojekten und Forschungsmethoden sowie die Befürchtung, solche Forschung würde von einem prinzipiell defizitären Lehr- und Unterrichtsmodell ausgehen.

Auf Akzeptanzprobleme stoßen Forschungen, insbesondere Einzelforschungen, aber genauso im wissenschaftlichen Diskurs. Dies hat unterschiedliche Gründe. Einer liegt darin, dass empirisches Arbeiten stets hinsichtlich Gegenstandswahl, -konstruktion und -operationalisierung anfechtbar ist. Zweifel an der Relevanz und Reichweite empirischer Einzelforschung hat wohl aber fachintern auch mit dem Paradoxon lehr-/lernwissenschaftlicher Forschung zu tun, aus der Beobachtung sowie Introspektion real ablaufender Lehr-/Lernprozesse und einer 
erfolgten Offenlegung dort vorgefundener Handlungen und Prozesse Empfehlungen für deren Weiterentwicklung und Veränderung abzuleiten, die selbst aber nicht mehr Gegenstand der Untersuchung sind - was wiederum in der oben erwähnten Vernachlässigung von empirischer Aktionsforschung und Wirkungsforschung begründet liegt. Dies hat Ursachen darin, dass Einzelforschungen stets einen begrenzten Wirklichkeitsausschnitt fokussieren müssen, sich aber nicht notwendigerweise ergänzen, sich mitunter widersprechen oder sogar ganz inkompatibel sind. Größere Forschungsverbünde konnten bislang aus Ressourcengründen kaum realisiert werden. Insbesondere Nachwuchswissenschaftler spüren diese Last und die Grenzen der eigenen Untersuchungen und regen immer wieder zur Bildung von regionalen und überregionalen Forschungsverbünden an. Wir sollten mehr auf sie hören.

\section{Forschungsinhärente Probleme}

Ich werde im Folgenden zwei exemplarische Bereiche ausführen, die allgemein Probleme für empirische Forschung im Kontext von Fremdsprachenunterricht bereiten und zu Enttäuschung bei Rezipienten solcher Forschung - auch bei wohlwollenden Fachwissenschaftlern und interessierten Praktikern - führen können. Es sind forschungsinhärente Probleme. Sie betreffen zum einen die grundsätzliche Konzeptualisierung von Lehren und Lernen in der Fremdsprachenforschung als Faktorenkomplexion und zum anderen die Möglichkeiten der Operationalisierung von Untersuchungsgegenständen im Rahmen forschungsmethodologisch begründeter Erhebungs- und Analyseansätze.

Zunächst zum Problem Faktorenkomplexion: Auf der einen Seite stimmen Erkenntnisse hinsichtlich der Mehrdimensionalität von Lernprozessen mit der Er- fahrung von Fremdsprachenlehrenden überein und sind daher für deren Professionsverständnis als wenig bedrohlich einzuschätzen. Auf der anderen Seite verhindern solche Forschungsergebnisse einfache Adaptionen von eher allgemein gehaltenen Handlungsempfehlungen. Denn was in einem spezifischen Kontext, der durch besondere Rahmenbedingungen und Lernende geprägt ist, angemessenes, empirisch begründetes fremdsprachenunterrichtliches Handeln darstellt, ist in einem anderen Kontext hinfällig, da dort andere Konstellationen herrschen. Es ist ein logisch unlösbares Dilemma, dass das Postulat der Faktorenkomplexion von Fremdsprachenlehr-/-lernprozessen eine generell eingeschränkte Übertragbarkeit und damit Generalisierbarkeit von Einzelforschung - sei sie hypothesentestend oder hypothesengenerierend - impliziert und folglich eine potenzielle Unendlichkeit von Forschung verursacht. Die im Rahmen von Forschungsprojekten unverzichtbare Auswahl (und damit der Ausschluss) von Variablen, die im Kontext spezifischer Lehr-/Lernszenarien und sämtlicher damit verbundener Bedingungen (Lehr-/ Lernmittel, Methoden etc.) erforscht werden, führt zu Ergebnissen, die nicht ohne Weiteres außerhalb des fokussierten Faktorenkomplexes und Wirklichkeitsausschnitts Aussagekraft besitzen. Die Annahme und Erfahrung von Faktorenkomplexion des Fremdsprachenunterrichts desavouiert somit gleichermaßen partikularistische Forschungsergebnisse, die erst durch diese Grundannahme überhaupt zustande kommen, aber genauso Forschungsergebnisse mit Allgemeingültigkeitsanspruch.

Diese Problematik wird besonders deutlich, wenn man die Beziehungen von Forschung im Bereich DaF und DaZ betrachtet. Lernumgebungen und Bedingungen für das Lehren und Lernen von 
DaZ unterscheiden sich massiv von denen für $\mathrm{DaF}$, so dass jeweils sorgfältig zu prüfen ist, $\mathrm{ob}$ in einem Bereich gewonnene Erkenntnisse für den anderen (zumindest teilweise) gültig sind oder ob sie überhaupt nicht auf den anderen Bereich übertragen werden können.

Es wäre vermessen, hierfür eine schnelle Lösung anbieten $\mathrm{zu}$ wollen. Ich wage aber dennoch $\mathrm{zu}$ behaupten, dass die Erforschung der tatsächlich in Lernszenarien vorliegenden Faktoren und ihrer Einflüsse auf das Fremdsprachenlernen noch lange nicht hinreichend erfolgt ist, um auszuschließen, dass durch den kritischen Vergleich vielfältiger Lernformen doch allgemeingültigere Aussagen inklusive Einschätzungen über Wirksamkeiten auf messbare Lernresultate möglich wären.

Weniger logische als ganz forschungspraktische Probleme bereitet die zugrunde gelegte Komplexität des Gegenstands Fremdsprachenlehren und -lernen bei der Operationalisierung von Untersuchungsgegenständen im Rahmen empirischer Untersuchungen. Spezifische Fragestellungen müssen untersuchungsintern angemessen ausgewählt werden, aber gleichzeitig die allgemeine Forschungsentwicklung im entsprechenden Gegenstandsbereich im Blick behalten. Bei jeder empirischen Studie stellen sich die Fragen neu, innerhalb welchen Untersuchungsdesigns welche Erkenntnisinteressen, Gegenstände, Datenerhebungs-, Datenaufbereitungs-, -analyse- und -interpretationsverfahren angemessen $\mathrm{zu}$ verbinden sind, wo geeignete und auskunftsbereite bzw. freiwillige Untersuchungsteilnehmer (Lehrende, Lernende) bzw. Lehr- und Lernkontexte zu finden sind und wie sich die Vorgehensweisen bei unvorhergesehenen Ereignissen während des Forschungsprozesses angemessen anpassen lassen. Gleichzeitig soll die forschungsmethodische Anlage der Stu- die auch Vergleichbarkeit mit anderen Untersuchungen ermöglichen. Und hier spiegelt sich die Problematik des Konstrukts Faktorenkomplexion in einer auch forschungsmethodologischen Auffassung von Fremdsprachenlehren und -lernen als dynamischen, komplexen und mehrdimensionalen, von unterschiedlichen Variablen konstituierten Untersuchungsgegenständen. Dieses hat Konsequenzen in den forschungsmethodologischen Entscheidungen, die von der Annahme eines komplexen Subjektmodells/Menschenbilds (vgl. Grotjahn 2005) über die umfassende Berücksichtigung affektiver Variablen sowie des kontextuellen (sozialen wie auch interaktiven) Lehr-/Lernumfelds inklusive der Präferenz von möglichst natürlichen Daten aus real ablaufenden Lehr-/Lernsituationen gegenüber künstlich elizitierten Daten bis hin zur Beachtung von Gütekriterien wie Gegenstandsangemessenheit, Nachvollziehbarkeit und Akzeptabilität im Forschungsprozess reichen (vgl. auch Arbeitsgruppe Fremdsprachenerwerb Bielefeld 1996).

\section{Schlussfolgerung}

Die oben beschriebenen Herausforderungen an empirische Forschung in unserem Bereich sind besonders brisant in einer Zeit, in der uns quantitative, outputorientierte Bildungsstudien zum Schulerfolg, Festsetzungen von Standards, Kerncurricula und Kompetenzen überrollen, die keine Pendants in fremdsprachenspezifischer, insbesondere spracherwerbsbezogener empirischer Forschung haben, ja ohne solche auszukommen glauben. Diese in der Öffentlichkeit stark beachteten und politisch enorm einflussreichen quantitativen Bildungsstudien messen Lernoutput und beschreiben Defizite, während empirische Fremdsprachenforschung - überspitzt ausgedrückt - noch Schwierigkeiten dabei hat, überhaupt zu 
bestimmen, wie man Lernzuwächse über längere Zeiträume umfassend, den Erwerbsmöglichkeiten entsprechend und natürlich auch reliabel und valide feststellen kann. Eine bessere Beteiligung in der empirischen Bildungsforschung wird nachhaltig dann am besten gelingen, wenn mehr Fremdsprachendidaktiker so qualifiziert werden, dass sie mit ausreichenden Kompetenzen in Forschungsmethoden (inkl. testmethodischer Kompetenzen) ausgestattet erfolgreich an solchen Forschungsprojekten mitarbeiten können. Hier stimmt mich zuversichtlich, dass sich in unserem Bereich die Testforschung stetig weiterentwickelt - auch als Folge der Entwicklung und Anwendung von C-Tests und TestDaF. $\mathrm{Zu}$ wünschen ist allerdings, dass fremdsprachenspezifische Testentwicklung und Testforschung noch stärker in engem Kontakt und im Verbund mit der Erforschung des Lehrens und Lernens von Fremdsprachen erfolgen und nicht zu bloßen Lieferanten von methodisch einwandfreien und überprüften Diagnoseinstrumenten mutieren, die für Selektionszwecke gebraucht, aber auch missbraucht werden können.

Hier muss langfristige Aufbauarbeit betrieben werden, die beginnen muss, indem eine forschungsmethodologische und -methodische Ausbildung der Studierenden in den Bachelor- und dann insbesondere in den Masterstudiengängen und in der Postgraduiertenausbildung Deutsch als Fremdsprache erfolgt. Eine solche Integration in die Studiengänge wurde in der Vergangenheit immer wieder gefordert, zuletzt z.B. von Grotjahn (2006) und Schlak (2004) - wann soll sie endlich realisiert werden? Schlak äußerte 2004 angesichts erster eingerichteter bzw. geplanter Bachelor- und Masterstudiengänge noch die Hoffnung, dass die forschungsmethodologische Grundausbildung bald zum festen Repertoire im Studienfach Deutsch als Fremdsprache gehören würde, und lieferte eine Reihe von bedenkenswerten Vorschlägen. Sie fanden bislang kaum Berücksichtigung. Ich befürchte, dass der heutige Trend sogar eher in eine andere Richtung geht. Viele DaF-/DaZ-Universitätsstandorte, die mit geringen Ressourcen ausgestattet sind, haben sich im Rahmen der Umsetzung des Bologna-Prozesses dafür entschieden, auf der Basis von Erfahrungen aus ehemaligen Nebenfach-, Aufbauund Zusatzstudiengängen anwendungsorientierte Masterstudiengänge $\mathrm{DaF} z u$ konzipieren, die v.a. Bachelorabsolventinnen und -absolventen der germanistischen Studiengänge eine überwiegend fremdsprachendidaktische Spezialisierung anbieten. Auch mittlere und gröBere Standorte für $\mathrm{DaF}$ (mit bis zu 4 Professuren) bieten bislang keine oder nur Ansätze einer systematischen forschungsmethodologischen Ausbildung an. Und nach ersten Erfahrungen mit dem neu eingerichteten Bielefelder DaFMasterstudiengang (und mit Blick auf die Methodenausbildung z. B. im Rahmen psychologischer oder soziologischer Studiengänge) komme ich zur vorläufigen Einschätzung, dass die Einrichtung ganzer Module mit mehr als einer in Forschungsmethoden einführenden Lehrveranstaltung und weitere Studienangebote, bei denen in konkreten Forschungsprojekten Methoden angewendet und erprobt werden, nötig sind, um Studierenden das Rüstzeug für selbstständig durchgeführte (kleinere) Projekte zu vermitteln. Es ist (noch) nicht zu spät, in den DaF-/DaZ-Studiengängen entscheidende Veränderungen vorzunehmen; der BAMA-Umstrukturierungsprozess ist im Bereich DaF/DaZ noch nicht abgeschlossen und für bereits eingerichtete Studiengänge können im Zuge der Evaluation und weiteren Entwicklung der Studiengänge Anpassungen schritt- 
weise vorgenommen werden, entsprechend vorhandenen Ressourcen und Kompetenzen. Wer soll aber diese Lehre anbieten, fragen sich jetzt vielleicht einige. Zweifellos wird es v. a. in der Aufbauphase immer wieder notwendig werden, für die Hochschullehre Kompetenz aus den Nachbarwissenschaften seinzukaufen ( z. B. durch die Investition von Mitteln zur Verbesserung der Lehre). Die Studienstandorte sollten Möglichkeiten standortübergreifender Konzepte entwickeln und sich gegenseitig unterstützen. Gesprächsbeiträge nach dem Vortrag dieses Beitrags auf der FaDaF-Jahrestagung regten z. B. die gemeinsame Entwicklung und Betreuung von E-Modulen an.

Zweifellos wird genauer zu differenzieren sein, welches forschungsmethodologische Wissen und Können Absolventinnen und Absolventen DaF-/DaZ-spezifischer Studiengänge benötigen. An dieser Stelle möchte ich erste Vorschläge unterbreiten. In den Bachelorstudiengängen sind Kompetenzen anzustreben, die die Rezeption von Forschung erlauben und an Praxisforschung heranführen (z. B. Aktionsforschung oder die Durchführung/Mitgestaltung von Evaluationen). Hierfür sind rezeptive und produktive Kenntnisse z. B. in folgenden Bereichen anzustreben: Erstellen und Beurteilen einfacher (Evaluations-)Instrumentarien, Konzeption und Bewertung von Fragebögen und Beobachtungsbögen, Durchführung von Leitfaden-Interviews und hierfür Aneignung von Interviewstrategien. Mit der Arbeit an der Entwicklung solcher Kompetenzen kann frühzeitig bereits in den ersten Studiensemestern begonnen werden, wobei auch komplexitätsreduzierte Einführungsliteratur eingesetzt werden kann (als exemplarisches Beispiel Moser 2003). Im Rahmen von Masterstudiengängen und in der Promotionsphase sollten Studierende auf die eigene Forschungspraxis vorbereitet wer- den, die im Rahmen von Master- und Doktorarbeiten selbstständig erfolgt. Hierfür sind Kompetenzen zu erarbeiten, z. B. in der begründeten Konzeption von Forschungsansätzen (qualitativ, quantitativ, gemischt) und der Auswahl geeigneter Datenerhebungsmethoden, z.B. die begründete Auswahl von Probanden oder die Zusammenstellung von Stichproben, z. B. Beachtung von Standards der Datenaufbereitung und Analyse (u. a. Transkriptionsverfahren, EDV-gestützte Verfahren der Analyse quantitativer und qualitativer Daten). Geeignete Fachliteratur ist verfügbar, auch das Volumen hat sich in den letzten Jahren insofern deutlich verbessert, dass einige Monographien zur Forschungsmethodik im Rahmen der Fremdsprachenforschung publiziert wurden, die teils einführenden, teils weiterführenden Charakter haben. Bezeichnenderweise sind dies fast ausnahmslos Publikationen in englischer Sprache (eine solche Ausnahme stellt Albert/Koster 2002 dar, vgl. aber auch die kritischen Anmerkungen von Grotjahn 2003), was trotz aller Rede von der internationalen Wissenschaftssprache Englisch für viele DaF-Studierende (darunter oft internationale Studierende) eine Hürde darstellt (exemplarische Beispiele: Brown 1988, Brown/Rodgers 2002, Dörnyei 2003, Ellis 2005, Gass/Mackey 2007, Mackey/Gass 2005, McDonough/McDonough 1997, Perry 2005, Seliger/Shohamy 1989).

Es ist mir ein besonderes Anliegen hervorzuheben, dass eine gute forschungsmethodische Ausbildung nicht allein für Nachwuchswissenschaftlerinnen und Nachwuchswissenschaftler und für die Aufrechterhaltung und Weiterentwicklung der universitären DaF-Standorte wichtig ist. Ich halte die kompetente Beherrschung des grundlegenden Handwerkszeugs für empirische Forschung für eine Schlüsselkompetenz jeder akade- 
misch ausgebildeten Kraft, die im Bereich der Vermittlung von Sprache und Kultur agiert. Nur wer über solche Grundlagen verfügt, wird Forschungsergebnisse angemessen rezipieren können (und wollen), z. B. das Kleingedruckte in statistischen Studien verstehen und solchen Werten wie $r, T, p, \alpha$ etc. Verfahren zuordnen können. Eine bessere Akzeptanz und fundiertere kritische Einschätzung von Forschungsergebnissen im Rahmen der professionellen Aus- und Weiterentwicklung kann dadurch gefördert werden. Des Weiteren kann eine bessere Befähigung zur methodisch kontrollierten Evaluation von Lehrangeboten und zu Aktionsforschung (siehe oben) das Professionsverständnis akademisch geschulter Sprachlehrkräfte weiterentwickeln und möglicherweise bei der weiteren Professionalisierung von Sprachlehre im Bereich DaF/DaZ nützlich sein. So betrachtet stellt Forschungskompetenz ein wichtiges Ausbildungsziel für die DaF-/DaZStudiengänge dar, die neben den (in alphabetischer Reihenfolge) didaktischmethodischen, kulturwissenschaftlichen, lehr-/lernwissenschaftlichen, literaturund sprachwissenschaftlichen Kompetenzen notwendigerweise anzustreben sind.

\section{Literatur}

Aguado, Karin; Riemer, Claudia: »Empirische Forschung: aus der Praxis für die Praxis?«. In: Wolff, Armin; Tanzer, Harald (Hrsg.): Sprache - Kultur - Politik. Regensburg: Fachverband Deutsch als Fremdsprache, 2000, 153-165 (Materialien Deutsch als Fremdsprache, 53).

Albert, Ruth; Koster, Cor J.: Empirie in Linguistik und Sprachlehrforschung. Ein methodologisches Arbeitsbuch. Tübingen: Narr, 2002.

Arbeitsgruppe Fremdsprachenerwerb Bielefeld: »Fremdsprachenerwerbsspezifische Forschung. Aber wie? Theoretische und methodologische Überlegungen«,
Deutsch als Fremdsprache 33 (1996), 144155; 200-210.

Bausch, Karl-Richard; Christ, Herbert; Krumm, Hans-Jürgen: »Fremdsprachendidaktik und Sprachlehrforschung «. In: Bausch, Karl-Richard; Christ, Herbert; Krumm, Hans-Jürgen (Hrsg.): Handbuch Fremdsprachenunterricht. Vierte, vollständig neu bearbeitete Auflage. Tübingen; Basel: Francke, 2003, 1-9.

Brown, James Dean: Understanding Research in Second Language Learning. A Teacher's Guide to Statistics and Research Design. Cambridge: Cambridge University Press, 1988.

Brown, James Dean; Rodgers, Theodore S.: Doing Second Language Research. Oxford: Oxford University Press, 2002.

Bundesministerium des Innern: Evaluation der Integrationskurse nach dem Zuwanderungsgesetz. Abschlussbericht und Gutachten über Verbesserungspotenziale bei der Umsetzung der Integrationskurse. Rambøll Management, Dezember 2006 [verfügbar unter: http:/ / www.bamf.de/cln_011/ nn_442016/SharedDocs/Anlagen/DE/ Integration/Downloads/Integrationskurse / Kurstraeger / Sonstiges / abschlussbericht-evaluation__IP, templateId=raw,property=publicationFile.pdf / abschlussbericht-evaluation_IP.pdf, 10.9.2007].

Caspari, Daniela: Fremdsprachenlehrerinnen und Fremdsprachenlehrer: Studien zu ihrem beruflichen Selbstverständnis. Tübingen: Narr, 2003.

Dörnyei, Zoltán: Questionnaires in Second Language Research. Construction, Administration, and Processing. Mahwah, NJ/ London: Lawrence Erlbaum, 2003.

Ellis, Rod; Barkhuizen: Analysing Learner Language. Oxford: Oxford University Press, 2005.

Fachverband Deutsch als Fremdsprache (2006): Grundsatzpapier des Fachverbands Deutsch als Fremdsprache (FaDaF) zur curricularen Basis der BA/MA-Studiengänge »Deutsch als Fremdsprache" (DaF) [verfügbar unter: http://www.fadaf.de/de/ daf_angebote/studieng_nge/grundsatzpapier.pdf, 10.9.2007].

Gass, Susan M.; Mackey, Alison: Data Elicitation for Second and Foreign Language Research. Mahwah, NJ/London: Lawrence Erlbaum, 2007. 
Grotjahn, Rüdiger: »On the methodological basis of introspective methods «. In: Faerch, Claus; Kasper, Gabriele (Hrsg.): Introspection in Second Language Research. Clevedon/Philadelphia: Multilingual Matters, 1987, 54-81.

Grotjahn, Rüdiger: »Rezensionsaufsatz. >Empirie in Linguistik und Sprachlehrforschung . Kritische Bemerkungen $\mathrm{zu}$ einem >methodologischen Arbeitsbuch ' ", Zeitschrift für Fremdsprachenforschung 14, 1 (2005a), 169-186.

Grotjahn, Rüdiger: »Subjektmodelle. Implikationen für die Theoriebildung und Forschungsmethodologie der Sprachlehrund Sprachlernforschung ", Zeitschrift für Fremdsprachenforschung 16, 1 (2005b), 2356.

Grotjahn, Rüdiger: »Zur Methodologie der Fremdsprachenerwerbsforschung «. In: Scherfer, Peter; Wolff, Dieter (Hrsg.): Vom Lehren und Lernen fremder Sprachen: Eine vorläufige Bestandsaufnahme. Berlin; Bern; Brüssel; Frankfurt a. M.; New York; Oxford; Wien: Lang, 2006, 247270.

Mackey, Alison; Gass, Susan M.: Second Language Research. Methodology and Design. Mahwah, NJ/London: Lawrence Erlbaum, 2005.

McDonough, Jo; McDonough, Steven: Research Methods for English Language Teachers. London: Arnold, 1997.
Moser, Heinz: Instrumentenkoffer für die Praxisforschung. Zürich: Verlag Pestalozzianum, 2003.

Perry, Fred L., Jr.: Research in Applied Linguistics. Becoming a Discerning Consumer. Mahwah, NJ/London: Lawrence Erlbaum, 2005.

Rankin, Jamie; Becker, Florian: »Does reading the research make a difference? A case study of teacher growth in FL German ", Modern Language Journal 90, 3 (2006), 353372.

Riemer, Claudia: »Für und über die eigene Unterrichtspraxis forschen: Anregungen zur Lehrerhandlungsforschung «. In: Schreiber, Rüdiger (Hrsg.): Deutsch als Fremdsprache am Studienkolleg. Unterrichtspraxis, Tests, Evaluation. Regensburg: Fachverband Deutsch als Fremdsprache, 2002, 129-143 (Materialien Deutsch als Fremdsprache, 63).

Schlak, Torsten: »Aus den Erfahrungen anderer Fächer lernen: Wege und Perspektiven der forschungsmethodologischen Ausbildung im Hochschulfach >Deutsch als Fremdsprache«", Info DaF 31, 5 (2004), 533-548.

Seliger, Herbert W.; Shohamy, Elana: Second Language Research Methods. Oxford: Oxford University Press, 1989.

Steinke, Ines: Kriterien qualitativer Forschung. Ansätze zur Bewertung qualitativempirischer Sozialforschung. Weinheim; München: Juventa, 1999. 